\title{
COSMOLOGICAL APPLICATION OF THE NEW GENERAL RELATIVITY
}

\author{
F.I. Mikhail* M.I. Wanas**, and G.G.L. Nashed* \\ * Mathematics Department, Faculty of Science, Ain Shams University, Cairo, Egypt \\ ** Astronomy Department, Faculty of Science, Cairo University, Giza, Egypt
}

The field equations of the new general relativity constructed by Hayashi and Shirafuji (1979), have been applied to two different geometric structures, given by Robertson (1932), in the domain of cosmology. In the first application a family of models, involving two of the parameters characterizing the field equations of the new general relativity, is obtained. In the second application the models obtained are found to involve one parameter only. The cosmological parameters in both applications are calculated and some cosmological problems are discussed in comparison with the corresponding results of other field theories. 


\section{Introduction}

Standard Big Bang model suffers from some difficulties. Several trials have been made to overcome these difficulties [1-3]. These trials can be classified into two classes. The first class considers the modification of the model itself [3]. The second class considers the modification of the general relativity (GR) [4-5]. In this respect there is a general belief that the geometrical structure upon which GR is based (Riemannian geometry), should be replaced by a more wider structure. Some authors believe that the absolute parallelism (AP) space is a good candidate for this purpose [6-11]. Some of these trials have been applied to cosmological problems [1-2], while others have not been applied, so far, in this domain.

It is the purpose of this paper to apply the new general relativity (NGR), constructed by Hayashi and Shirafuji (1979), in the domain of cosmology and to compare the obtained results with those of the previous theories. In section 2 we give a brief review of the geometric structure upon which the NGR based on it. In section 3, we are going to give the two tetrad spaces satisfying the cosmological principle, i.e, the homogeneity and isotropy of the universe, constructed by Robertson (1932), to apply them to the field equations of the NGR. In section 4 a brief review of the NGR is discussed. In section 5, application of the field equations of the NGR to the two tetrad spaces given in section 3 . In section 6 we compare the results with different field theories based on the AP-space.

\section{AP-Spaces}

The AP-space is a 4-dimensional vector space, in which every point is labelled by a set of 4 -independent variables $x^{\nu}(\nu=0,1,2,3)$. We associate with each point of this space 4-linearly independent contravariant vectors denoted by $\lambda_{i}^{\mu}$ where $(\mu=0,1,2,3)$ indicates the coordinate component and $(i=0,1,2,3)$ indicates the vector number. Each component of $\lambda_{i}^{\mu}$ is assumed to be a singal-valued function of $x^{\nu}$ and differentiable to any required order. The normalized cofactor of $\lambda_{i}^{\mu}$ in the determinate $\lambda=\left\|\lambda_{i}^{\mu}\right\| \neq 0$ is denoted by $\underset{i \mu}{\lambda}$ and is defined by $\underset{i \mu}{\lambda}=\frac{1}{\lambda}$ (cofactor of ${ }_{i}^{\lambda^{\mu}}$ on $\left.\left\|\lambda_{i}^{\mu}\right\|\right)$. It can be easily shown that $\lambda_{i \mu}$ are covariant components of the tetrad and

$$
\begin{aligned}
& { }_{i}^{\lambda_{i}^{\mu}}{ }_{i \nu}=\delta^{\mu}{ }_{\nu}, \\
& { }_{i \mu} \lambda_{k}{ }^{\mu}=\delta_{i k},
\end{aligned}
$$

where $\delta^{\mu}{ }_{\nu}$ are the ordinary Kronecker delta. Greek indices (world) are used to indicate the coordinate components and are written in a covariant or contravariant position, i.e, 
$\lambda_{i}^{\mu}$ or $\lambda_{i \mu}$. Latin indices are used to indicate the number of the vector which are written in a lower position only and are called "mesh indices". A summation convention will be applied to the mesh indices as well as to the world one. Using the tetrad vector, we can define the following second order symmetric tensor:

$$
\begin{aligned}
& g_{\mu \nu}=\underset{i \mu}{\lambda_{\mu}} \underset{\nu}{\lambda}, \\
& g^{\mu \nu}=\underset{i}{\lambda^{\mu}}{\underset{i}{\nu}}^{\nu},
\end{aligned}
$$

thus, $g_{\mu \nu} g^{\nu \sigma}=\delta^{\mu}{ }_{\sigma}$.

It has been shown [12] that $(g)^{1 / 2}=\lambda$ where $g=\left\|g_{\mu \nu}\right\|$. We can always define a Riemannian space, associated with the AP-space, by taking the symmetric tensor (2.3) to play the role of the metric tensor, viz.

$$
d s^{2}=g_{\mu \nu} d x^{\mu} d x^{\nu}
$$

It is obvious from (2.5) that the associated Riemannian space will have a +ve definite metric. To compare the results of any theory, based on the AP-space, with the results of GR, the metric of the associated Riemannian space should have the Lorentz signature (indefinite). In this case the metric will take the form:

$$
d s^{2}=g_{\mu \nu}^{*} d x^{\mu} d x^{\nu}
$$

where $g^{*}{ }_{\mu \nu}=e_{i} \lambda_{i \mu} \lambda_{i \nu}, e_{i}$ is the Levi-Civita's indicator $(-1,+1,+1,+1)$. Some authors, instead of using Lorentz indicator $e_{i}$ in their applications, prefer to insert Lorentz signature by multiplying the zeroth vector by $\left(i=(-1)^{1 / 2}\right)$ [1]. The symmetric tensor (2.3) and its conjugate (2.4) can be used to raise and lower world indices in the usual manner.

In the AP-space we can define two different connections:

(i) A symmetric connection $\left\{\begin{array}{l}\alpha \\ \mu \nu\end{array}\right\}$ defined in terms of the symmetric tensor $g_{\mu \nu}$ given by (2.3) in the standard way, i.e,

$$
\left\{\begin{array}{l}
\alpha \\
\mu \nu
\end{array}\right\}=\frac{1}{2} g^{\sigma \alpha}\left(g_{\mu \sigma, \nu}+g_{\nu \sigma, \mu}-g_{\mu \nu, \sigma}\right) .
$$

(ii) A non symmetric connection $\Gamma^{\alpha}{ }_{\mu \nu}$ defined in terms of the tetrad vectors directly as a consequence of the AP-condition [12

$$
\Gamma_{\mu \nu}^{\alpha}=\lambda_{i}^{\alpha}{ }_{i \mu, \nu}=-\lambda_{i \mu i} \lambda^{\alpha}, \nu
$$

Consequently, three different types of the absolute derivatives can be defined:

$$
A_{; \nu}^{\mu}=A_{, \nu}^{\mu}+\left\{\begin{array}{l}
\mu \\
\alpha \nu
\end{array}\right\} A^{\alpha},
$$




$$
\begin{aligned}
& A_{+\mid \nu}^{\mu}=A_{, \nu}^{\mu}+\Gamma^{\mu}{ }_{\alpha \nu} A^{\alpha}, \\
& A_{-\mid \nu}^{\mu}=A_{, \nu}^{\mu}+\Gamma_{\nu \alpha}^{\mu} A^{\alpha},
\end{aligned}
$$

where $A^{\mu}{ }_{, \nu}=\frac{\partial A^{\mu}}{\partial x^{\nu}}$. We can define the following third order tensors:

$$
\begin{aligned}
\Lambda^{\alpha}{ }_{\mu \nu} & =\Gamma_{\mu \nu}^{\alpha}-\Gamma_{\nu \mu}^{\alpha}=-\Lambda_{\nu \mu}^{\alpha}, \\
\gamma^{\alpha}{ }_{\mu \nu} & =\Gamma_{\mu \nu}^{\alpha}-\left\{\begin{array}{l}
\alpha \\
\mu \nu
\end{array}\right\} \\
\Delta^{\alpha}{ }_{\mu \nu} & =\gamma^{\alpha}{ }_{\mu \nu}+\gamma^{\alpha}{ }_{\nu \mu}
\end{aligned}
$$

It can be easily shown that [12, 10]

$$
\begin{gathered}
\gamma_{\mu \nu}^{\alpha}=\lambda_{i}^{\alpha} \lambda_{i \mu ; \nu}=-\lambda_{i \mu} \lambda_{i}{ }^{\alpha}{ }_{; \nu}, \\
\gamma_{\alpha \mu \nu}=-\gamma_{\mu \alpha \nu} .
\end{gathered}
$$

It is clear that the skew part and the symmetric part of $\Gamma^{\alpha}{ }_{\mu \nu}$ and $\gamma^{\alpha}{ }_{\mu \nu}$ are respectively given by

$$
\begin{gathered}
\Gamma_{[\mu \nu]}^{\alpha}=\gamma_{[\mu \nu]}^{\alpha}=\frac{1}{2} \Lambda_{\mu \nu}^{\alpha}, \\
\gamma_{(\mu \nu)}^{\alpha}=\frac{1}{2} \Delta^{\alpha}{ }_{\mu \nu} .
\end{gathered}
$$

The tensor $\Lambda^{\alpha}{ }_{\mu \nu}$ is called the torsion tensor and $\gamma^{\alpha}{ }_{\mu \nu}$ is the contortion tensor. Both can be related by

$$
\gamma_{\alpha \mu \nu}=\frac{1}{2}\left(\Lambda_{\alpha \mu \nu}+\Lambda_{\nu \mu \alpha}-\Lambda_{\mu \alpha \nu}\right) .
$$

Contracting any one of the three tensors (2.12) by setting $\alpha=\nu$ we get the basic vector

$$
C_{\mu}=\Lambda_{\mu \alpha}^{\alpha}=\gamma_{\mu \alpha}^{\alpha}=\Delta_{\mu \alpha}^{\alpha}
$$

This is the same vector denoted by Hayashi and Shirafuji [11] by $v_{\mu}$ with a negative sign. They introduced another third order tensor given by:

$$
t_{\alpha \mu \nu}=\frac{1}{2}\left(\Lambda_{\alpha \mu \nu}+\Lambda_{\mu \alpha \nu}\right)-\frac{1}{6}\left(g_{\nu \alpha} C_{\mu}+g_{\mu \nu} C_{\alpha}\right)+\frac{1}{3} g_{\alpha \mu} C_{\nu}
$$

and the axial vector

$$
a_{\mu}=\frac{1}{6} \epsilon_{\mu \nu \rho \sigma} \Lambda^{\nu \rho \sigma}
$$

where

$$
\epsilon_{\mu \nu \rho \sigma}=\sqrt{-g} \delta_{\mu \nu \rho \sigma}
$$


with $\delta_{\mu \nu \rho \sigma}$ completely skew symmetric and normalized as $\delta_{0123}=-1$. The tensor defined by (2.19) has the following properties:

$$
\begin{aligned}
t_{\alpha \mu \nu} & =t_{\mu \alpha \nu}, \\
g^{\mu \nu} t_{\alpha \mu \nu} & =0, \\
t_{\alpha \mu \nu}+t_{\mu \nu \alpha}+t_{\nu \alpha \mu} & =0 .
\end{aligned}
$$

\section{Geometric Structures}

In order to examine the NGR, with respect to the cosmological problem, we are going to use the two tetrad spaces given by Robertson 13 and satisfying the cosmological principle (homogeneity and isotropy of the universe). The two structures have the same associated Riemannian space characterized by the well known Robertson-Walker metric. These two geometric structures are defined by the following tetrads (written in the spherical polar coordinate, i.e., $\left.x^{0}=t, x^{1}=r, x^{2}=\theta, x^{3}=\phi\right)$ viz

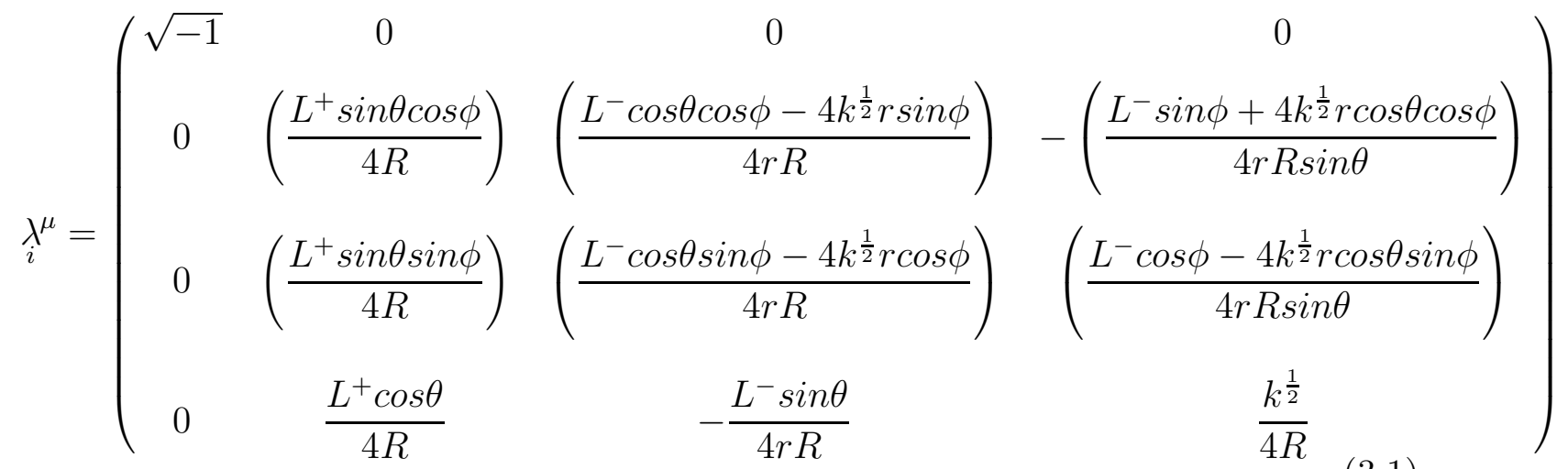

and

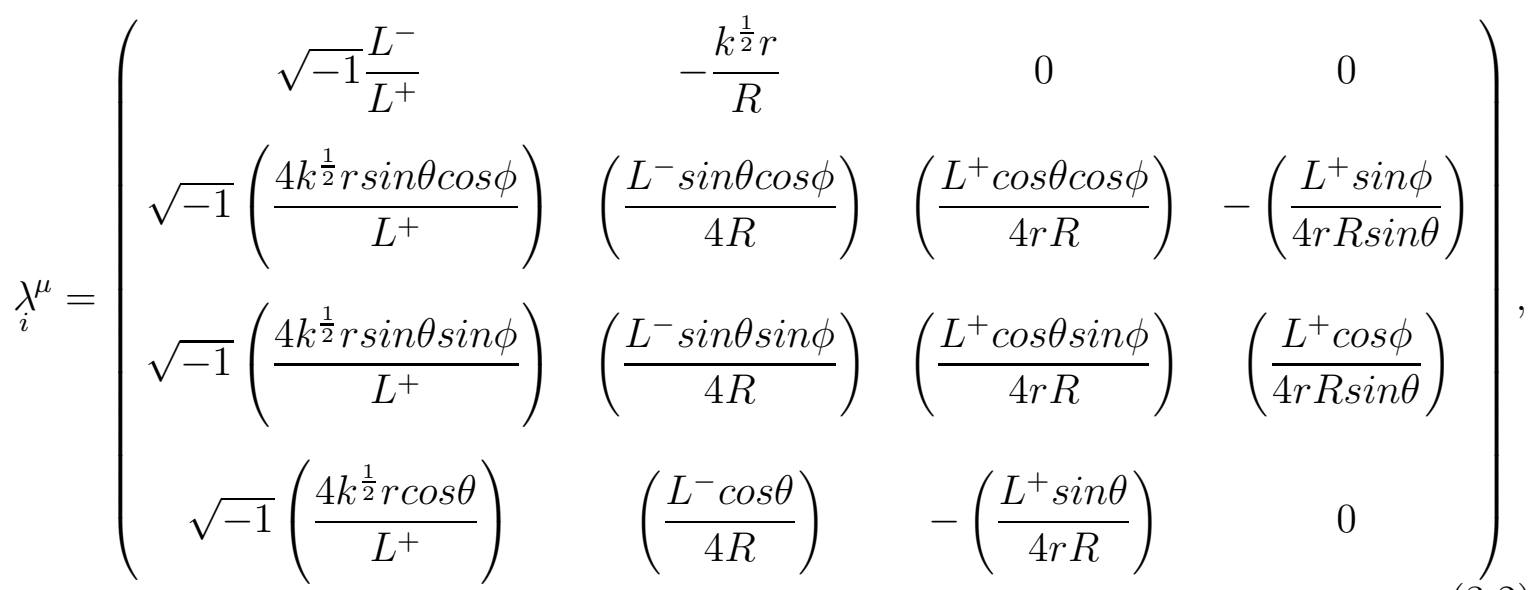


where $L^{ \pm}=4 \pm k r^{2}, \mathrm{k}$ is the curvature constant $(k=-1,0,+1)$, and $\mathrm{R}(\mathrm{t})$ is an unknown function of $t$. The Riemannian space associated with the tetrads (3.1), (3.2) will have the same Robertson-Walker metric:

$$
d s^{2}=-d t^{2}+\frac{16 R^{2}(t)}{L^{+2}}\left(d r^{2}+r^{2}\left(d \theta^{2}+\sin ^{2} \theta d \phi^{2}\right)\right)
$$




\section{Hayashi-Shirafuji Theory}

In 1979 Hayashi and Shirafuji constructed a theory which they called "New General Relativity", NGR. They have used an AP-space for its formulation, and a variational principle to derive its field equations. They were able to derive a set of field equations involving three different parameters. They took the Lagrangain density in the form

$$
\mathcal{L}=\frac{\lambda}{2 \kappa}\left[R+2 \bar{d}_{1}\left(t^{\mu \nu \lambda} t_{\mu \nu \lambda}\right)+2 \bar{d}_{2}\left(v^{\mu} v_{\mu}\right)+2 \bar{d}_{3}\left(a^{\mu} a_{\mu}\right)\right]
$$

where $\bar{d}_{1}, \bar{d}_{2}$, and $\bar{d}_{3}$ are dimensionless parameters of the theory. They have chosen their Lagrangian such that it will be invariant under the following:

a) the group of general coordinate transformations,

b) the group of global Lorentz transformations,

c) the parity operation, i.e., ${\underset{0}{\mu}}^{\mu} \rightarrow \underset{0}{\lambda^{\mu}}$, and $\underset{a}{\lambda^{\mu}} \rightarrow-{\underset{a}{\mu}}^{\mu}$, where $a=1,2,3, \mathrm{R}$ is the Ricci scalar tensor and $t_{\alpha \mu \nu}, C_{\mu}, a_{\mu}$, are the tensors defined in section 2. By applying a variational principle to the above Lagrangian they [11] were able to obtained the field equations in the form:

$$
G^{\mu \nu}+2 \kappa D_{\lambda} F^{\mu \nu \lambda}-2 \kappa C_{\lambda} F^{\mu \nu \lambda}+2 \kappa H^{\mu \nu}-\kappa g^{\mu \nu} L^{\prime}=\kappa T^{\mu \nu},
$$

where $G^{\mu \nu}$ is the Einstein tensor defined by

$$
G^{\mu \nu}=R^{\mu \nu}-\frac{1}{2} g^{\mu \nu} R
$$

with $R^{\mu \nu}$ being the Ricci tensor, and

$$
\begin{aligned}
F^{\mu \nu \lambda} & =\frac{1}{\kappa}\left[\bar{d}_{1}\left(t^{\mu \nu \lambda}-t^{\mu \lambda \nu}\right)-\bar{d}_{2}\left(g^{\mu \nu} C^{\lambda}-g^{\mu \lambda} C^{\nu}\right)-\frac{\bar{d}_{3}}{3} \epsilon^{\mu \nu \lambda \rho} a_{\rho}\right]=-F^{\mu \lambda \nu}, \\
H^{\mu \nu} & =\Lambda^{\rho \sigma \mu} F_{\rho \sigma}{ }^{\nu}-\frac{1}{2} \Lambda^{\nu \rho \sigma} F_{\rho \sigma}^{\mu}=H^{\nu \mu} \\
L^{\prime} & =\frac{\mathcal{L}}{\lambda} \\
T^{\mu \nu} & =\frac{1}{\lambda} \frac{\delta \mathcal{L}_{M}}{\delta b^{k}{ }_{\nu}} b^{k \mu}
\end{aligned}
$$

Here $\mathcal{L}_{M}$ denotes the Lagrangian density of material fields and $T^{\mu \nu}$ is the material energy-momentum tensor which is nonsymmetric in general.

It is well known that the conservation law in GR is given by

$$
T_{G R}^{(\mu \nu)}{ }_{; \nu}=0
$$

where $T_{G R}{ }^{(\mu \nu)}$ is the symmetric material energy-momentum tensor of GR and semicolon denotes the covariant derivative with respect to the Christoffel symbol. This law does 
not follow from (4.2), however. Instead, they [11] derive the response equation

$$
T_{; \nu}^{\mu \nu}=\gamma^{\nu \lambda \mu} T_{[\nu \lambda]}
$$

The antisymmetric part $T^{[\mu \nu]}$ is due to the contribution from the intrinsic spin of fundamental spin-1/2 particles. For macroscopic test particles employed in terrestrial experiments, effects due to intrinsic spin can be ignored, and hence their energy-momentum tensor can be supposed to be symmetric and satisfy (4.8). Accordingly, the equation of motion for macroscopic test particles is the geodesic equation of the metric.

\section{Cosmological Models}

In the present section we are going to apply (4.2) together with the response equation (4.9) to the two geometric structures given in section 3. In order to compare the models that will arise from the NGR with observation we are going to use the following parameters [14].

The Hubble's parameter, $H_{0}=\frac{\dot{R}\left(t_{0}\right)}{R\left(t_{0}\right)}=\frac{\dot{R}_{0}}{R_{0}}=100 h_{0} k m s^{-1} M p c^{-1}$, where $0.5 \leq h \leq 1$,

the deceleration parameter, $q_{0}=-\frac{\ddot{R}_{0}}{R_{0}} H_{0}{ }^{-2}$,

the denisty parameter, $\sigma_{0}=\frac{\rho_{0}}{\rho_{c}}$,

where $\rho_{c}$ is the critical denisty defined by, $\rho_{c}=3 H^{2} / \kappa$ [14, $\rho_{0}$ is the proper denisty given by the model, $\kappa$ is the Einstein constant ( $=8 \pi$ in relativistic units), and the dot denotes differentiation with respect to the time t. For the present application we are going to use the material energy tensor of the perfect fluid with the following non-vanishing components

$$
T_{0}^{0}=\rho_{0}, \quad T_{1}^{1}=T_{2}^{2}=T_{3}^{3}=-p_{0} .
$$

In the dust case the equation of the state will be

$$
p_{0}=0
$$

In the case of radiation, the equation of state will be

$$
p_{0}=\frac{1}{3} \rho_{0}
$$

\subsection{MODELS RESULTING FROM THE FIRST STRUCTURE.}

Applying the field equations (4.2) to the tetrad (3.1), we have found that the skew part of the L.H.S. of (4.2) is identically zero, hence $T^{[\mu \nu]}=0$. In this case the field 
equations (4.2) will give rise to the two differential equations:

$$
\begin{aligned}
Y \frac{\dot{R}^{2}}{R^{2}}+B \frac{k}{R^{2}} & =\frac{\kappa}{3} \rho_{0}, \\
2 Y \frac{\ddot{R}}{R}+Y \frac{\dot{R}^{2}}{R^{2}}+B \frac{k}{R^{2}} & =-\kappa p_{0},
\end{aligned}
$$

where the new parameters $\mathrm{Y}$ and $\mathrm{B}$ are given by $Y=\left(1-3 \bar{d}_{2}\right), B=\left(1+4 / 3 \bar{d}_{3}\right)$. Also since $T^{[\mu \nu]}=0$, the general law of the response equation of NGR will reduce to the standard conservation law of GR giving rise in the present application, to

$$
R \frac{d \rho_{0}}{d R}=-3\left(\rho_{0}+p_{0}\right)
$$

The solutions of (5.4) and (5.5) in the two cases of the physical interest given by (5.2) and (5.3) are summarized in the following table

Table(I) Solution of the field equations

\begin{tabular}{|c|c|c|}
\hline Curvature Constant & Dust Case & Radiation Case \\
\hline$k=+1$ & $R=\beta(1-\cos w) \quad t=\left(\frac{Y}{B}\right)^{\frac{1}{2}} \beta(w-\sin w)$ & $R(t)=\frac{\left(\gamma^{2}-\left[t \delta^{2}-\gamma^{2}\right]^{2}\right)^{\frac{1}{2}}}{\delta}$ \\
\hline$k=0$ & $R(t)=\left(\frac{9 B \beta}{2 Y}\right)^{\frac{1}{3}} t^{\frac{2}{3}}$ & $R(t)=(2 \gamma)^{\frac{1}{2}} t^{\frac{1}{2}}$ \\
\hline$k=-1$ & $R=\beta(\cosh w-1) t=\left(\frac{Y}{B}\right)^{\frac{1}{2}} \beta(\sinh w-w)$ & $R(t)=\frac{\left(\left[t \delta^{2}+\gamma^{2}\right]^{2}-\gamma^{2}\right)^{\frac{1}{2}}}{\delta}$ \\
\hline
\end{tabular}

where $\gamma^{2}=\kappa M / 3 Y, \delta^{2}=B / Y, \beta=4 \pi N / 3 B$, M and $\mathrm{N}$ being constants of integration resulting from (5.5). These solutions involve the two parameters $\bar{d}_{2}, \bar{d}_{3}$ characterizing the field equations of the NGR.

Using the definitions of the parameters listed above we get the following values for the solutions given in table I 
Table(II) Summary of The Cosmological Parameters

\begin{tabular}{|c|c|c|c|}
\hline \multicolumn{2}{|c|}{ Parameters } & Dust Case & Radiation Case \\
\hline \multirow{5}{*}{$q_{0}$} & $k-\perp 1$ & 1 & $\gamma^{2}$ \\
\hline & $\kappa=+1$ & $1+\cos w$ & $\overline{\left(t \delta^{2}-\gamma^{2}\right)^{2}}$ \\
\hline & $k=0$ & $\frac{1}{a}$ & 1 \\
\hline & 1 & $\frac{2}{1}$ & $\gamma^{2}$ \\
\hline & $k=-1$ & $\overline{1+\cosh w}$ & $\overline{\left(t \delta^{2}+\gamma^{2}\right)^{2}}$ \\
\hline \multirow{5}{*}{$H_{0}$} & & $(B)^{1 / 2} \quad \sin w$ & $(\gamma-t)$ \\
\hline & $\kappa=+1$ & $(\bar{Y}) \quad \overline{R_{0}(1-\cos w)^{2}}$ & $\overline{\left(2 t \gamma-t^{2}\right)}$ \\
\hline & $k=0$ & $\frac{2}{3} t_{0}^{-1}$ & $\frac{1}{2} t_{0}^{-1}$ \\
\hline & $k=-1$ & $(\underline{B})^{1 / 2} \frac{\sinh w}{}$ & $(\gamma+t)$ \\
\hline & & $(\bar{Y}) \quad \overline{R_{0}(\cosh w-1)^{2}}$ & $\overline{\left(2 t \gamma+t^{2}\right)}$ \\
\hline \multirow{5}{*}{$\sigma_{0}$} & & $2 Y$ & $\gamma^{2} Y$ \\
\hline & & $1+\cos w$ & $\overline{2\left(t \delta^{2}-\gamma^{2}\right)^{2}}$ \\
\hline & $k=0$ & $\mathrm{Y}$ & $\frac{Y}{2}$ \\
\hline & 1 & $2 Y$ & $\gamma^{2} Y$ \\
\hline & & $\overline{1+\cosh w}$ & $\overline{2\left(t \delta^{2}+\gamma^{2}\right)^{2}}$ \\
\hline
\end{tabular}

Now we are going to discuss some defects of the above models

\section{particles horizon.}

We are going to examine whether or not the resulting world models involve particle horizones. To do so we have to recall the metric of the Riemannian space associated with these models as given by (3.3), i.e.,

$$
d s^{2}=-d t^{2}+\frac{16 R^{2}(t)}{L^{+2}}\left[d r^{2}+r^{2}\left(d \theta^{2}+\sin ^{2} \theta d \phi^{2}\right)\right]
$$

according to this metric, the coordinate radial distance of any particle of the model from the origin of coordinates is given by

$$
r=\int \frac{d r}{4+k r^{2}}
$$

hence the proper distance of this particle from the origine is given by

$$
S=4 R_{0} r=4 R_{0} \int \frac{d r}{4+k r^{2}}
$$

To find out the limiting proper distance up to which observations can be carried out, we note that for a radial ray of light, we get

$$
\int_{t_{1}}^{t 0} \frac{d t}{4 R}=\int_{0}^{r} \frac{d r}{4+k r^{2}}
$$


$\underline{\text { Fork }=+1:}$

$$
r=2 \tan \left[\left(\frac{Y}{B}\right)^{\frac{1}{2}} \frac{\left(w_{0}-w_{1}\right)}{2}\right],
$$

where $w_{0}=\cos ^{-1}\left\{\left(1-q_{0}\right) / q_{0}\right\}, w_{1}=\cos ^{-1}\left\{1-\left(2 q_{0}-1\right) / q_{0}(1+Z)\right\}$. We can see from (5.10), that $\mathrm{r}$ has a finite value if $Z \rightarrow \infty$. For simplicity we call this finite value as $r_{f}$ and integrate (5.8) from 0 to $r_{f}$, we finally get

$$
S=2 R_{0} \tan ^{-1} \frac{r_{f}}{2} \quad \text { for } \quad k=+1
$$

Similarly we can get

$$
\begin{aligned}
& S=R_{0} r_{f} \quad \text { for } \quad k=0, \\
& S=2 R_{0} \tanh ^{-1} \frac{r_{f}}{2} \quad \text { for } \quad k=-1,
\end{aligned}
$$

from (5.4) and the definition of the parameters we can get the relation

$$
2 q_{0}-1=\frac{k}{{R_{0}^{2} H_{0}^{2}}^{2}} \frac{B}{Y}=\frac{k q_{0} R_{0}}{\beta},
$$

which will give rise to

$$
R_{0}=\left(\frac{B}{Y}\right)^{\frac{1}{2}} \frac{1}{H_{0}} \sqrt{\frac{k}{\left(2 q_{0}-1\right)}} \quad \text { for } \quad k= \pm 1
$$

and for $k=0, R_{0}$ is given by

$$
R_{0}=\left(\frac{9 B \beta}{2 Y}\right)^{\frac{1}{3}} t_{0}^{\frac{2}{3}}
$$

We see from (5.11), and (5.12) that $\mathrm{S}$ has a finite value as $Z \rightarrow \infty$ in the three cases. This means that all the above models contain particle horizons. However, it may be of interest to point out that as $Y \rightarrow 0$, i.e., $\kappa \bar{d}_{2} \rightarrow \frac{1}{3}$, the horizon will be extended further and further and $S \rightarrow \infty$. This value is calculated in the case of dust. It can be shown that the same calculations could be drawn in the case of radiation.

Flatness problem.

¿From (5.4) we have

$$
\frac{\dot{R^{2}}}{R^{2}}=\frac{\kappa}{3 Y} \rho_{0}-\delta^{2} \frac{k}{R^{2}}
$$

which will give rise to

$$
\frac{\sigma_{0}-1}{\sigma_{0}}=\frac{3 B}{\kappa} \frac{k}{\rho_{0} R^{2}}
$$


it was shown [15]

$$
\frac{\sigma_{0}-1}{\sigma_{0}}= \begin{cases}10^{-15} B & \text { at } 1 \mathrm{sec} . \\ 10^{-49} B & \text { at } 10^{-35} \text { sec. } \\ 10^{-57} B & \text { at } 10^{-43} \text { sec. }\end{cases}
$$

It is clear from (5.18) that $\sigma_{0} \sim 1$ when $k \sim 0$ and this means that the above models suffer from the flatness problem.

\subsection{MODLES RESULTING FROM THE SECOND STRUCTURE.}

Applying the field equations (4.2) to the tetrad (3.2), and using the same procedure applied in the first application we get

$$
\begin{aligned}
\left(\frac{\dot{R}^{2}}{R^{2}}+\frac{k}{R^{2}}\right) Y & =\frac{\kappa}{3} \rho_{0}, \\
\left(\frac{2 \ddot{R}}{R}+\frac{\dot{R}^{2}}{R^{2}}+\frac{k}{R^{2}}\right) Y & =-\kappa p_{0},
\end{aligned}
$$

the solution of equation (5.19), in the cases mentioned by (5.2), (5.3) can be summarized in the following table

Table(III) Solution of the field equations

\begin{tabular}{|c|c|c|}
\hline Curvature Constant & Dust Case & Radiation Case \\
\hline$k=+1$ & $R=\alpha(1-\cos w) \quad t=\alpha(w-\sin w)$ & $R(t)=\left(2 \gamma t-t^{2}\right)^{\frac{1}{2}}$ \\
\hline$k=0$ & $R(t)=\left(\frac{9 \alpha}{2 Y}\right)^{\frac{1}{3}} t^{\frac{2}{3}}$ & $R(t)=(2 \gamma)^{\frac{1}{2}} t^{\frac{1}{2}}$ \\
\hline$k=-1$ & $R=\alpha(\cosh w-1) \quad t=\alpha(\sinh w-w)$ & $R(t)=\left(2 \gamma t+t^{2}\right)^{\frac{1}{2}}$ \\
\hline
\end{tabular}

where $\alpha=4 \pi / 3 Y$. These solutions involve only one parameter $\overline{d_{2}}$, characterizing the field equations of NGR.

Using the definitions of the parameters listed above we get the following values for the solutions given in table IV 
Table(IV) Summary of The Cosmological Parameters

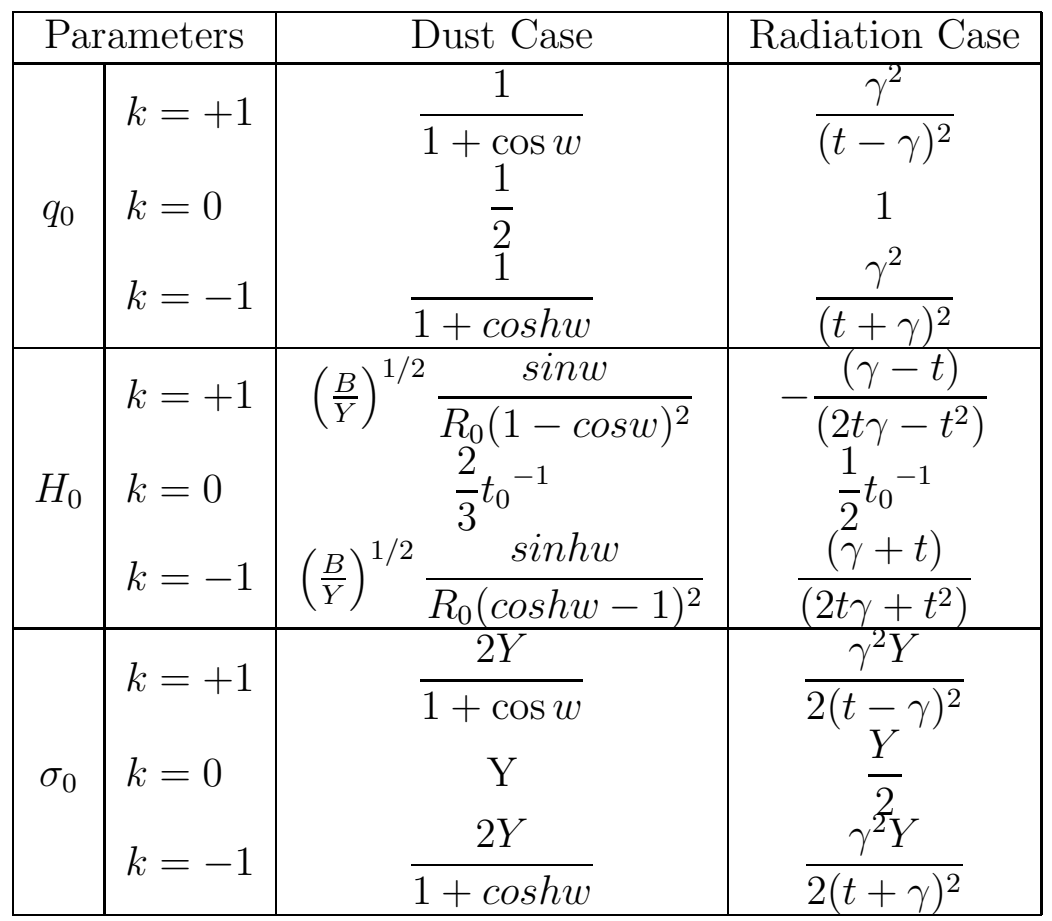

We would like to point out that the models obtained here different from those of GR. However, the models obtained from the second structure will reduce to those of GR if $Y \rightarrow 0$.

\section{particles horizon.}

By the same procedure applied in the subsection 5.1 we can evaluate the proper distance for the present structure (in the case of dust)

$$
\begin{aligned}
& S=R_{0} \tan ^{-1} \frac{r_{f}}{2} \quad \text { for } \quad k=+1, \\
& S=R_{0} r_{f} \quad \text { for } \quad k=0, \\
& S=R_{0} \tanh ^{-1} \frac{r_{f}}{2} \quad \text { for } \quad k=-1,
\end{aligned}
$$

from (5.19) and the definition of the parameters we can get the relation

$$
2 q_{0}-1=\frac{k}{R_{0}^{2} H_{0}^{2}}=\frac{k q_{0} R_{0}}{\alpha}
$$

which will give rise to

$$
R_{0}=\frac{1}{H_{0}} \sqrt{\frac{k}{\left(2 q_{0}-1\right)}} \quad \text { for } \quad k= \pm 1
$$


and for $k=0, R_{0}$ is given by

$$
R_{0}=\left(\frac{9 \alpha}{2 Y}\right)^{\frac{1}{3}} t_{0}{ }^{\frac{2}{3}}
$$

We see from (5.20) that $\mathrm{S}$ has a finite value as $Z \rightarrow \infty$ in the three cases. This means that all the above models contain particle horizons.

Also all the above models of the second application suffer from the flatness problem. Singularity problem.

$\overline{\text { It clear from table (II) }}$ and (IV) that $H_{0}$ tends to infinity as $t \rightarrow 0$ in all the cases and this means that the denisty will be infinite. This means that the two applications suffer from the singularity problem.

\section{Comparison With Other Field Theories.}

Now we are going to compare the resultes of the present work with the results of other field theories, constructed using AP-space, to show the difference between them in the cosmological application.

Table(V) Comparison With Other Theories

\begin{tabular}{|c|c|c|c|c|}
\hline Criterion & GR (1916) & GFT(1977) & MTT (1978) & NGR (1979) \\
\hline Space-time & $\begin{array}{c}\text { Riemannian } \\
\text { Space }\end{array}$ & AP-Space & AP-Space & AP-Space \\
\hline Free Parameters & No & No & One & Three \\
\hline Energy-Momentum & Symmetric Tensor & $\begin{array}{c}\text { Symmetric } \\
\text { Geometric } \\
\text { Tensor } T^{\mu \nu}\end{array}$ & $\begin{array}{c}\text { Symmetric } \\
\text { Phenomol- } \\
\text { ogical Tensor }\end{array}$ & $\begin{array}{c}\text { Non-Symmetric } \\
\text { Phenomological } \\
\text { Tensor }\end{array}$ \\
\hline $\begin{array}{c}\text { Basic } \\
\text { Assumption }\end{array}$ & $\begin{array}{c}\text { Homogeneity } \\
\text { and Isotropy }\end{array}$ & $\begin{array}{c}\text { Homogeneity } \\
\text { and Isotropy }\end{array}$ & $\begin{array}{c}\text { Homogeneity } \\
\text { and Isotropy }\end{array}$ & $\begin{array}{c}\text { Homogeneity } \\
\text { and Isotropy }\end{array}$ \\
\hline $\begin{array}{c}\text { Need for an } \\
\text { Equation of State }\end{array}$ & Yes & No & Yes & Yes \\
\hline $\begin{array}{c}\text { Curvature Constant } \\
\text { for Non-Static } \\
\text { Non-Empty Model }\end{array}$ & $+1,0,-1$ & -1 & $+1,0,-1$ & $+1,0,-1$ \\
\hline $\begin{array}{c}\text { Number of } \\
\text { Allowed Models }\end{array}$ & Many & One & Many & Many \\
\hline $\begin{array}{c}\text { Theory } \\
\text { Indicator for }\end{array}$ & Macroscopic & Macroscopic & Macroscopic & Macroscopic and \\
Strong Field & Singularity & $\Lambda$ & Singularity & Singularity \\
\hline Particle Horizon & Yes & No & Yes & Conditional \\
\hline $\begin{array}{c}\text { Flatness Problem } \\
\text { Singularity Problem }\end{array}$ & Yes & No & Yes & Yes \\
\hline \multirow{2}{*}{ Yes } & Yes & Yes \\
\hline
\end{tabular}

Where $\Lambda=\frac{1}{2}(\sigma-\bar{\omega}), \bar{\omega}=\gamma_{\mu \epsilon}^{\sigma} \gamma_{\sigma \nu}^{\epsilon}-\gamma_{\nu \epsilon}^{\sigma} \gamma_{\sigma \mu}^{\epsilon}, \sigma_{\mu \nu}=\gamma_{\epsilon \mu}^{\sigma} \gamma_{\sigma \nu}^{\epsilon}$ 


\section{References}

[1] Sáez, D., and De Juan, T. (1984), GRG, $\underline{16}, 5$.

[2] Wanas, M.I. (1989), Astrophys. Space Sci. , 154165.

[3] Lind, A.D. (1990), "Inflation and Quantum Cosmology" Acadmic Press, Inc.

[4] Hoyle, F. (1948), M. N. R. A. S. , 108, 372.

[5] Hoyle, F. (1949), M. N. R. A. S. , 109, 365.

[6] Einstein, A. (1929), Sitz. der Preuss. Aksd. der wiss. , $1,2$.

[7] Levi-Civita, T. (1950), "A simplified presentation of Einstien's unified field equations" London: Blackie and Son.

[8] Mikhail, F.I. (1964), Al Nuovo Cimento Series X, $\underline{32}$, P. 886.

[9] Mikhail, F.I., and Wanas, M.I. (1977), Proc. Roy. Soc., Lond. A $\underline{356}, 471$.

[10] Mфller, C. (1978), Mat. Fys. Medd. Dan. Vid. Selsk. , 39, 13.

[11] Hayashi, K., and Shirafuji, T. (1979) Phys. Rev. D. , 19, 3524.

[12] Mikhail, F.I. (1962), Ain Shams Sci. Bull. , $\underline{6}, 87$.

[13] Robertson, H.P. (1932), Ann. Math. Princeton, 33, 496.

[14] Narlikar, J.V. (1983), "Introduction to Cosmology", Janes and Barlett. Boston.

[15] Turner, M.S. (1987), GRG, 11. 